VoL. 51 (1995) [517-520]

\title{
A GENERALISATION OF AHLFORS-SCHWARZ LEMMA TO RIEMANNIAN GEOMETRY
}

\author{
KoK Seng Chua
}

\begin{abstract}
Our main result shows that a conformal mapping of hyperbolic $n$-space into another Riemannian manifold with scalar curvature bounded above by $-n(n-1)$ is necessarily distance decreasing. This is a generalisation of Ahlfors' version of the Schwarz-Pick lemma to Riemannian Geometry.
\end{abstract}

\section{INTRODUCTION}

Pick's formulation of the classical Schwarz lemma (see [2]) in terms of the Poincarè metric is well known in complex analysis. In a seminal paper [1], Ahlfors extended the Schwarz-Pick lemma and established a connection between curvature and hyperbolicity. Since then there have been many important generalisations of Ahlfors's result (see for example $[3,4,6])$. However it appears to us that all of these generalisations are to holomorphic maps between higher dimensional complex manifolds. In this note, we replace the holomorphy assumption by conformality and prove a direct generalisation of the Ahlfors-Schwarz lemma to higher dimensional Riemannian manifolds. Our main result is the following:

THEOREM 1. Let $(M, g)$ be a Riemannian manifold of dimension $n \geqslant 2$ and scalar curvature $\leqslant-n(n-1)$. Let $f:\left(H^{n}, g_{0}\right) \rightarrow(M, g)$ be a smooth mapping from the hyperbolic $n$-space into $M$ which is conformal away from the critical points of $f$. Then $f$ is distance decreasing, that is, $f^{*} g \leqslant g_{0}$.

We note that by choosing $n=2$ and letting $f$ be an analytic function into a Riemann surface, we obtain as an immediate corollary of Theorem 1 Ahlfors' version of the Schwarz lemma (Theorem $A$ in [1]).

\section{MAIN RESULTS}

Before proving our main theorem, we consider first the following compact version of our result which seems to be of some independent interest:

Received 25 August 1994

Copyright Clearance Centre, Inc. Serial-fee code: 0004-9729/95 \$A2.00+0.00. 
Lemma 1. Let $(M, g)$ be a compact Riemannian manifold of dimension $n \geqslant 2$. Let $\widehat{g}=e^{2 \varphi} g$ be a conformal metric such that scal $(\widehat{g}) \leqslant \operatorname{scal}(g)<0$ where scal is the scalar curvature. Then $\widehat{g} \leqslant g$. (That is, $\varphi \leqslant 0$.)

Proof: We shall denote by $k$ the quantity $\operatorname{scal}(g) / n(n-1)$ and likewise for $\hat{k}$. A direct computation then shows that (see for example [7])

$$
e^{2 \varphi} \widehat{k}=k-\frac{2}{n} \Delta \varphi-\frac{n-2}{n}|\nabla \varphi|^{2}
$$

where the metric dependent quantities above are computed with respect to $g$. Since $-k>0$ and $\hat{k} / k \geqslant 1$, we have from (1) that

$$
\begin{aligned}
\frac{2}{n} \Delta \varphi+\frac{n-2}{n}|\nabla \varphi|^{2} & =(-k)\left(e^{2 \varphi} \frac{\hat{k}}{k}-1\right) \\
& \geqslant(-k)\left(e^{2 \varphi}-1\right) .
\end{aligned}
$$

By compactness of $M$, we can choose a point $x$ such that $\varphi$ attains a maximum. Then

$$
\Delta \varphi(x) \leqslant 0 \quad \text { and } \quad \nabla \varphi(x)=0 .
$$

(2) and (3) imply

$$
0 \geqslant \frac{2}{n} \Delta \varphi(x) \geqslant(-k)\left(e^{2 \varphi(x)}-1\right)
$$

so that $e^{2 \varphi} \leqslant e^{2 \varphi(x)} \leqslant 1$.

We shall now prove our main theorem by generalising the argument in [2].

Proof of Theorem 1: We shall let $\widehat{g}=f * g=e^{2 \varphi} g_{0}$ which is defined except at the critical points of $f$. We need to prove $\varphi \leqslant 0$. We note first that we only need consider regular points of $f$ since $\varphi$ must approach $-\infty$ whenever $d f$ is singular. We shall take $H^{n}$ to be the unit ball $\left\{x \in \mathbf{R}^{n}:|x|<1\right\}$ and

$$
\left(g_{0}\right)_{i j}=\rho \delta_{i j} \quad \rho=\frac{4}{\left(1-|x|^{2}\right)^{2}}
$$

Since $\operatorname{scal}(\widehat{g})=\operatorname{scal}(g) \circ f \leqslant-n(n-1)$, we have $\hat{k} \leqslant-1$ and $k=-1$ so that again $\widehat{k} / k \geqslant 1$. (1) and (2) follow where the metric dependent quantities are now with respect to $g_{0}$. The same argument as in the proof of Lemma 1 shows that $\varphi(x) \leqslant 0$ at any interior maximum point $x$ of $\varphi$. It suffices for us to consider the boundary behaviour. 
We shall suppose first the $\widehat{g}$ is such that $\varphi$ has a $C^{2}$ extension to the closed ball. Using (5) and the usual formulae for conformal change of metric (see [7]), we can rewrite (2) as

$$
\frac{2}{n \rho} \tilde{\Delta} \varphi+\frac{n-2}{n \rho^{2}}\langle\tilde{\nabla} \rho, \tilde{\nabla} \varphi\rangle+\frac{n-2}{\tilde{\nabla} \rho}|\tilde{\nabla} \varphi|^{2} \geqslant e^{2 \varphi}-1
$$

where the tilde quantities are with respect to the Euclidean metric. In terms of $x$ (6) can be rewritten as

$$
\begin{aligned}
& \left(1-|x|^{2}\right)^{2} \tilde{\Delta} \varphi+2(n-2)\left(1-|x|^{2}\right)\langle x, \tilde{\nabla} \varphi\rangle \\
& \quad+\frac{(n-2)}{2}\left(1-|x|^{2}\right)^{2}|\widetilde{\nabla} \varphi|^{2} \geqslant 2 n\left(e^{2 \varphi}-1\right) .
\end{aligned}
$$

Letting $|x| \rightarrow 1$, we have again $e^{2 \varphi(x)} \leqslant 1$ since clearly the left hand side of (7) approaches 0 by our smoothness assumption on $\widehat{g}$. It follows that we have $e^{2 \varphi} \leqslant 1$ everywhere in $B^{n}$. For general $\widehat{g}$, we set $\widehat{g}_{r}(x)=r^{2} \widehat{g}(r x)(0<r<1)$. Then $\widehat{g}_{r}$ satisfies the same smoothness condition above and $\widehat{k}_{r}(x)=\widehat{k}(r x) \leqslant-1$ so that we have $\widehat{g}_{r} \leqslant g$ by what we have proved above. Letting $r$ tend to 1 gives $\hat{g} \leqslant g$ by continuity.

It seems that the assumption in Theorem 1 that the metric be hyperbolic is too strong. All we need in the proof is that the metric should blow up suitably at the boundary which in our case is ensured by completeness of the hyperbolic metric. It thus appears to us that the following more general result (which includes both Theorem 1 and Lemma 1) should be true.

CONJECTURE. Let $(M, g)$ be a complete Riemannian manifold of dimension $n \geqslant 2$. If $\widehat{g}=e^{2 \varphi} g$ is a conformal metric such that $\operatorname{scal}(\widehat{g}) \leqslant \operatorname{scal}(g)<0$, then $\widehat{g} \leqslant g$.

We note that Ahlfors' version of the Schwarz lemma has been used to give geometric proofs of many classical results in complex analysis (see [5]). It is clear that some of these can be generalised to higher dimension using our Theorem 1 . We hope to come back to this later. We only note here the following immediate consequence.

COROLLARY. A conformal self map of the unit ball is an isometry of hyperbolic space.

\section{REFERENCES}

[1] L.V. Ahlfors, 'An extension of Schwarz's lemma', Trans. Amer. Math. Soc. 43 (1938), 359-364.

[2] L.V. Ahlfors, Conformal invariants: Topics in geometric function theory (McGraw-Hill, New York, 1973). 
[3] S.S. Chern, 'On holomorphic mappings of Hermitian manifolds of the same dimension', in Proceedings Symposium Pure Mathematics 11 (Amer. Math. Soc., Providence, R.I., 1968), pp. 157-170.

[4] H. Grauert and H. Reckziegel, 'Hermitsche Metriken und normale Familien holomorpher Abbildungen', Math. Z. 89 (1965), 108-125.

[5] D. Minda and G. Schober, 'Another elementary approach to the theorems of Landau, Montel, Picard and Schottky', Complex Variables Theory Appl. 2 (1983), 157-164.

[6] S.T. Yau, 'A general Schwarz lemma for Kahler manifolds', Amer. J. Math. 100 (1978), 197-203.

[7] K. Yano and M. Obata, 'Conformal changes of Riemannian metrics', J. Differential Geom. 4 (1970), 53-72.

14 Cornwall Gardens

Singapore 1026 